\title{
Análise estatística para avaliação do comportamento de parâmetros hidráulicos aplicada a rede real de topografia montanhosa
}

\section{Statistical analysis to evaluate hydraulic parameters behavior applied to real mountain topography network}

Data de entrada: 24/07/2019

Data de aprovação: $29 / 01 / 2020$

Luane Tais da Silva Vieira' ${ }^{1}$ Fernando das Graças Braga da Silva ${ }^{1 *}$ | André Carlos da Silva ${ }^{1}$ |

Luiz Felipe Silva ${ }^{1}$

DOI: https://doi.org/10.36659/dae.2021.044

ORCID ID

Vieira LTS (D) https://orcid.org/0000-0001-9582-6179

Silva FGB iD https://orcid.org/0000-0002-3803-2257
Silva AC (iD) https://orcid.org/0000-0002-8415-700X

Silva LF (iD https://orcid.org/0000-0002-3174-9984

\section{Resumo}

O monitoramento de parâmetros hidráulicos em campo normalmente tem custo elevado. Em redes de distribuição de água, a compreensão é extremamente importante para a tomada de decisões que visem ao acesso a melhor eficiência hídrica e energética, principalmente relacionadas a operação e controle de perdas de água. $\mathrm{O}$ objetivo do trabalho foi utilizar ferramentas estatísticas para análise de setor real de distribuição de água do Sul de Minas Gerais. A metodologia fundamentou-se em análises estatísticas que possibilitam interpretar informações para subsidiar a tomada de decisões futuras, entre elas o Teste t de Student. A partir de análises de adaptação de um simples modelo matemático, foram avaliadas as perdas por nós a partir dos dados tratados. Concluiu-se que pode-se extrair muita informação de uma rede real pelas análises; aqui especificamente são contempladas particularidades de região montanhosa.

Palavras-chave: Parâmetros hidráulicos. Sistema de distribuição de água e análise estatística.

\section{Abstract}

Monitoring hydraulic parameters in the field is usually costly. Understanding water distribution networks is extremely important for decision making aimed at better access to water and energy efficiency, especially related to water loss operation and control. The objective of this work was to use statistical tools for real sector analysis of water distribution in southern Minas Gerais. The methodology was based on statistical analysis that make it possible to interpret information to support future decision making. As a result of several statistical analysis, such as the t test. From analysis from adaptation of a simple mathematical model we analyzed the losses from the treated data. We concluded that much information can be extracted from a real network by the analysis, here specifically for mountainous region particularities.

Keywords: Hydraulic parameters. Water distribution system and statistical analysis.

\footnotetext{
${ }^{1}$ Universidade Federal de Itajubá - Itajubá - Minas Gerais - Brasil.

*Autor correspondente: ffbraga.silvaagmail.com.
} 


\section{INTRODUÇÃO}

Atualmente tem-se observado problemas relacionados ao abastecimento de grandes centros urbanos associados ao aumento da população, diretamente ligado ao consumo, que acabam contribuindo para redução de disponibilidade de água em qualidade e quantidade. Tal assunto tornou-se um tema de preocupação mundial. Nesse contexto, as redes de distribuição de água como sistemas hídricos enfrentam problemas de grandes perdas de água.

Um agravante é que as redes de água existentes são em geral antigas e ineficientes, com tubulações e conexões que necessitam de manutenção frequente e cara. Outro fator são as altas pressões no sistema de distribuição de água, que provocam o rompimento das tubulações e causam grandes vazamentos. Dessa forma, na maioria dos casos a gestão de pressão é uma das estratégias de gestão de fugas (FONTANA; GIUGNI; PORTOLANO, 2012).

Por se tratar de obras enterradas, muitas vezes os sistemas de distribuição de água são esquecidos (MUTIKANGA; SHARMA; VAIRAVAMOORTHY, 2013). Segundo Fontana, Giugni e Portolano (2012), as perdas nas redes chegam a ultrapassar de 30 a 40\%. A implantação de um projeto de rede de água deveria balancear o custo de implantação geralmente baixo, com o custo da manutenção, que acaba sendo alto e frequente (PINNTO et al., 2017). Segundo Kanakoudis et al. (2013), as perdas podem ser divididas em dois tipos: as aparentes (não físicas) e as reais (físicas).

As análises de parâmetros hidráulicos são essenciais na avaliação do funcionamento das redes de distribuição de água, sendo também a forma mais eficiente de prever o comportamento do sistema quando ocorrem alterações das situações normais da rede. Essas análises em geral são feitas em vazão, pressão, rugosidade, perdas de carga nos trechos, entre outros, e suas altera- ções podem ocorrer devido a vazamentos, quebra, envelhecimento de tubos, válvulas e registros com defeito ou fora da vida útil (FORMIGA; CHAUDHRY, 2008).

Vários métodos para análise hidráulica dos sistemas de distribuição foram criados e aperfeiçoados, pois apresentam a solução para diversos tipos de problemas relacionados à rede. Lamoglia (2013) utilizou em seu trabalho o modelo de simulação hidráulica EPANET para avaliar o sistema de distribuição de água de São Lourenço, MG, observando o comportamento da rede e determinando os valores de perdas de água com base em vazão e pressão e o custo desse volume perdido. Ribeiro (2014) propôs a calibração da rede de abastecimento dessa mesma rede, empregando um modelo proposto por Silva (2003) com o uso do algoritmo genético com o objetivo de determinar a eficiência hídrica e energética do sistema. Santos (2016) estudou um modelo de calibração para ajuste de parâmetros de rugosidade, demanda e vazamento. Bobsin (2014) estudou a rede com o objetivo de examinar as particularidades e o comportamento de demanda. Fortes (2016) analisou as perdas de água e relações energéticas utilizando metodologia de balanço.

Segundo Vieira (2011) e Reis (2015), a análise estatística de dados é uma excelente ferramenta para diagnóstico de uma rede. Por meio dela é possível trabalhar dados coletados e observados, de forma a facilitar a compreensão dos resultados e a validação das informações descritas. Ao final da análise, as ações escolhidas como solução para o problema dependerão em parte dos dados coletados.

A utilização de software de análise dados estatísticos vem com uma proposta de facilitar e melhorar os resultados obtidos pelos programas de diagnósticos dos dados. Esses softwares possuem eficácia e facilidade de uso, além de dimi- 
nuir o tempo levado para execução dos cálculos e conclusões dos resultados.

Guidi (2016) analisou a variação de consumo de água utilizando dados obtidos por sistemas remotos no município de Franca (SP). 0 objetivo do trabalho foi determinar valores de $\mathrm{K} 1$ (coeficiente de vazão máxima diária) e K2 (coeficiente de máxima vazão horária), comparando com valores existentes na literatura. Já Melo (2014), em seu trabalho, aplicou técnica de análise estatística na avaliação de desempenho e confiabilidade de estações de tratamento de água (ETA).

Cambrainha e Fontana (2015) estudaram a análise da aplicação de investimentos em perdas no Nordeste brasileiro, com o objetivo de avaliar o quanto o investimento no setor de água é refletido na redução das perdas. Guedes et al. (2012) realizaram em seu trabalho a aplicação da análise estatística multivariada no estudo da qualidade da água do Rio Pomba (MG), com o objetivo de identificar os grupos de poluição presentes.

Rodrigues (2014) avaliou em seu trabalho o impacto da utilização de diferentes equipamentos de medição no índice de perdas em um setor isolado do sistema de abastecimento de água da cidade de Campo Grande (MS), para determinar qual seria o melhor tipo de medidor para cada sistema e melhor opção de hidrômetro para cada consumidor.

Deste modo, conclui-se que os trabalhos hidráulicos com análises estatísticas não são frequentes na literatura.

\section{OBJETIVO}

Os objetivos específicos do artigo são os relacionados a seguir:

- Aplicar metodologia de análise estatística em um sistema;
- Extrair conhecimentos para melhor conhecimento do sistema hídrico em questão.

\section{MATERIAIS E MÉTODOS}

A metodologia a ser utilizada neste trabalho consiste em análise estatística de parâmetros hidráulicos de pressão de uma rede de abastecimento de água.

A pesquisa se inicia pela seleção da rede de estudo com base em dados obtidos em pesquisas feitas em 2014 pelo NUMMARH - Núcleo de Modelagem e Simulação em Meio Ambiente e Recursos e Sistemas Hídricos da Universidade Federal de Itajubá. Em seguida foi feita a análise de dados e informações para identificar quais são válidas para a pesquisa atual. A partir daí foi definida qual cidade do sul de Minas Gerais teve a rede estudada, levando em consideração os trabalhos já realizados pelo grupo anteriormente. Essa engloba três bairros: Palmela, Moradas da Serra e Vale dos Pinheiros e pertence a um setor isolado (SANTOS, 2016). E tem como companhia responsável pelo abastecimento de água e esgoto da cidade a SAAE (serviço autônomo de água e esgoto).

Os dados foram coletados do ano de 2014 , entre os dias 20 e 28 de setembro, pois esse ano foi o último de coleta de dados. Durante esses sete dias de 15 em $15 \mathrm{~min}$ foram marcados os valores de pressão registrados na rede e posteriormente elaborado um banco de dados. A rede possui dados de 19 loggers de pressão; deles, 18 foram monitorados e 15 nós considerados válidos, três foram descartados por apresentar erros de medição.

Os pontos monitorados são demonstrados na Fig. 1: 


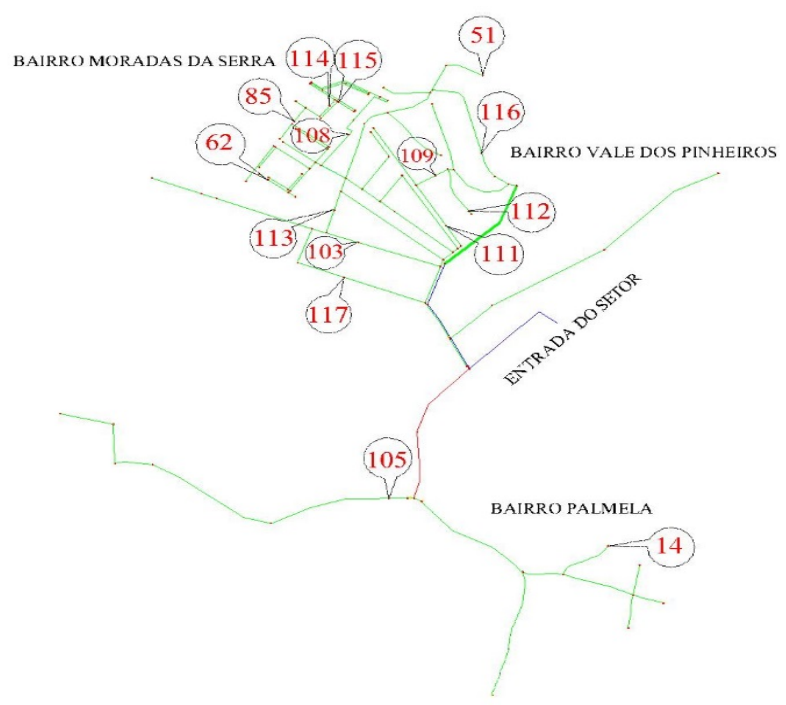

Figura 1 - Pontos de monitoramento de 7 dias

Fonte: Santos (2016)

Para analisar os dados, foram utilizadas planiIhas eletrônicas e softwares de apoio para análises gráficas. Ao escolher essa ferramenta, foram determinados quais seriam os dados que se desejava coletar; inicialmente foram analisados os parâmetros estatísticos de média, desvio padrão, pressão máxima e mínima e os percentis. Posteriormente foi realizado o Teste $t$ de Student para comparação de médias obtidas entre duas populações distribuídas normalmente. Os valores das médias foram dispostos em uma matriz para o procedimento de aplicação do Teste t, identificando diferenças significativas entre os valores, ou seja, valor de p menor ou igual a 0,05. As diferenças significativas, ou seja, valores de $p$ abaixo ou igual a 0,05 foram discutidas, e analisados os possíveis motivos pelos quais apresentam esse resultado.

Como para a rede estudada temos valores de pressão, decidiu-se calcular a porcentagem de perdas relativa ao valor de pressão obtido. Utilizou-se uma fórmula para cálculo das perdas na rede de estudo baseado nos trabalhos de Tucciarelli, Criminisi e Termini (1999) e na de Silva (2003), sendo dada por:
$\Sigma$ perdas $=\mathrm{P}_{\text {média }} 0,5 \cdot 7,27$

Onde: Perdas são representadas em percentual e a pressão média (Pmédia) em mca.

Foram identificados os limites de pressão para as perdas mínimas de $35 \%$ e máxima de $65 \%$, baseado no trabalho de Fortes (2016) ajustado para o setor em estudo onde perda real na rede é de $65,40 \%$. Para isso calculou-se de maneira inversa: conhecido o valor de perda, foi calculada inversamente a pressão máxima e mínima para os limites admitidos. 0 coeficiente 0,5 foi baseado em pesquisas anteriores e o valor 7,27 foi encontrado por método de tentativa e erro.

\section{RESULTADOS E DISCUSSÃO}

Os pontos também foram comparados por setor para compreender como funciona cada região. O primeiro ponto estudado é a entrada da rede, analisando dados de vazão e velocidade. A entrada da rede fica localizada no Solar dos Lagos. Foi feita análise de média, desvio padrão e variância, e os resultados são expressos na Tabela 1. 
Foram considerados 15 pontos de medidas para o cálculo de média, desvio padrão e variância referente a esta tabela.

As análises foram resumidas a partir do trabalho de Vieira (2019). Assim como nos pontos (Nós), o menor valor de média não teve diferença expres- siva em relação aos demais dias da semana, sendo o menor valor obtido em uma segunda-feira e o maior no dia 24/09, quarta-feira. Nota-se que a vazão é menor nos finais de semana e durante a semana vai aumentando gradativamente até quarta-feira e depois diminuindo até sábado, sendo menor aos domingos.

Tabela 1 - Análise dos dados de vazão Solar dos Lagos

\begin{tabular}{|c|c|c|c|}
\hline \multicolumn{4}{|c|}{ Vazão (L/S) } \\
\hline Dia & Média & Desvio Padrão & Variância \\
\hline $20 / 09 / 2014$ & 10,361 & 0,844 & 0,713 \\
\hline $21 / 09 / 2014$ & 9,905 & 1,415 & 2,004 \\
\hline $22 / 09 / 2014$ & 11,360 & 2,742 & 7,517 \\
\hline $23 / 09 / 2014$ & 11,255 & 2,742 & 7,521 \\
\hline $24 / 09 / 2014$ & 11,545 & 2,514 & 6,322 \\
\hline $25 / 09 / 2014$ & 11,362 & 2,862 & 8,192 \\
\hline $26 / 09 / 2014$ & 11,019 & 2,220 & 4,928 \\
\hline $27 / 09 / 2014$ & 10,755 & 1,965 & 3,862 \\
\hline $28 / 09 / 2014$ & 10,457 & 1,920 & 3,686 \\
\hline $29 / 09 / 2014$ & 9,431 & 2,446 & 5,981 \\
\hline
\end{tabular}

Fonte: Autor

O menor valor de desvio padrão foi apresentado no dia 20/09, sábado, quando os valores de vazões variaram menos que nos demais dias em relação à média. Do dia 22/09 ao 26/09, os valores de desvio padrão foram bem similares, ficando entre 2,22 e 2,86 mca. Assim, conclui-se que quanto maior é a vazão média mais alto também é seu valor de desvio padrão.

Foi gerado um gráfico com valores de pressão e consumo por setor da rede de estudo, para que se pudesse entender como os bairros influenciam a rede total. A Fig. 2 evidencia os resultados obtidos.

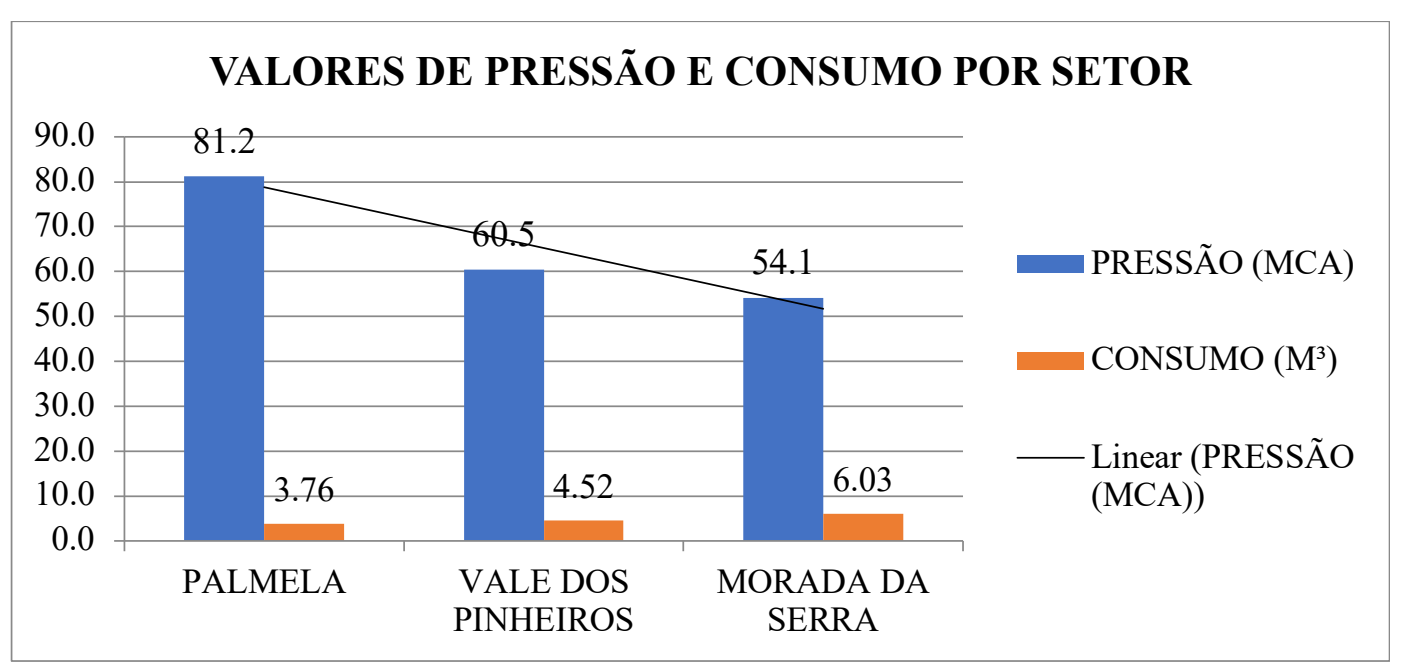

Figura 2 - Pressão e consumo por setor. Fonte: Autor 
O Bairro Vale dos Pinheiros possui uma parte com cotas altimétrica mais baixa e outra mais alta; a média de pressão nesse bairro fica entre os dois outros bairros, com valor de 60,5 mca, e sua média de consumo é de 4,52 l/s também entre o bairro Palmela com 3,76 l/s e o bairro Morada da Serra com 6,03 l/s. A característica que justifica esse valor de consumo possivelmente é que o bairro possui valor aquisitivo maior que o Bairro Palmela e menor que o Morada da Serra, o que implica no consumo da população.

Caracterizado o valor de pressão por setor, foi feito um gráfico com valor de média por nó. Assim, obtemos os nós com valores mais altos e os mais baixos, esses resultados são apresentados na Fig. 3 .

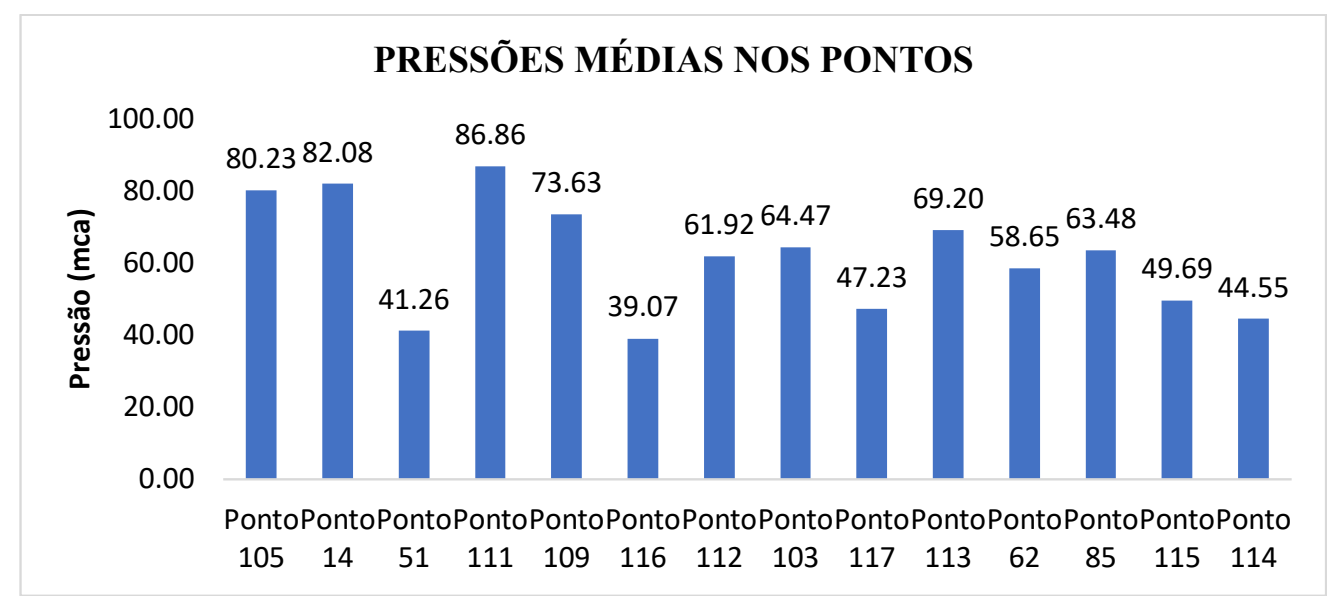

Figura 3 - Gráfico das médias por Nó. Fonte: Autor

Nesse gráfico pode-se observar também que as pressões em sua maior parte ficam acima do valor de $50 \mathrm{mca}$, que seria o valor ideal de pressão para uma rede. Abaixo de 50 mca ficaram cinco valores, entre eles o Nó 114 e o 115, pertencentes ao bairro morada da Serra, e os Nós 51, 111 e 117, pertencentes ao bairro Vale dos Pinheiros.

Na Tabela 2 foram calculados os parâmetros estatísticos por bairros que compõem a rede. Os dados foram coletados durante os sete dias da semana, assim podemos compará-los. O bairro Palmela possui média de pressão maior que os outros dois, sendo seu valor de $81,2 \mathrm{mca}$, e o bairro Morada da Serra apresentou valor mais baixo, de 54,1 mca, pois se encontra totalmente em uma área mais alta da rede, o que diminui a pressão na tubulação.

O maior valor de desvio padrão foi do bairro Vale dos Pinheiros, 16,8 mca. Essa região possui algumas diferenças de níveis, o que acaba influenciando nas pressões, tornando umas mais elevadas que as outras. A pressão máxima foi no bairro Vale dos Pinheiros, sendo 86,9 mca e o mínimo também nesse bairro, 39,1 mca. Isso ocorre possivelmente devido a diferenças de cotas altimétricas dos pontos de coleta de dados.

Tabela 2 - Dados estatísticos por setor, calculados durantes os 7 dias.

\begin{tabular}{|c|c|c|c|c|c|c|c|}
\hline & Média & Dv Pad & Mediana & P90\% & P10\% & Pmax \\
\hline Palmela & 81,2 & 1,3 & 81,2 & 80,4 & 81,9 & 82,1 \\
\hline Vale Dos Pinheiros & 60,5 & 16,8 & 63,2 & 40,6 & 77,6 & 86,9 \\
\hline Morada Da Serra & 54,1 & 8,5 & 54,2 & 46,1 & 62,0 & 63,5 \\
\hline
\end{tabular}


A Tabela 3 apresenta, para uma melhor compreensão, os valores das médias da pressão na rede, destacando as diferenças que foram consideradas significativas.

Os cálculos foram feitos para todos os Nós, dos dias 20/09/2014 a 27/09/2014. Os resultados foram os seguintes:

Quando comparados os valores do dia 20/09 e os demais dias, podemos notar que os valores ficaram abaixo 0,05 . Isso pode ter ocorrido porque o dia 20/09 foi o primeiro dia de coleta e o equipamento se encontrava em adaptação aos locais de medição, assim seus valores médios acabam sendo diferentes dos demais dias, que possuem mais dados válidos. Nos outros dias praticamente não foram observadas diferenças significativas. Possivelmente tal achado se deve em razão de os valores de pressão não terem tido grandes oscilações e a média.

O Nó 14 apresentou resultados diferentes em relação ao Nó 105. Separando as diferenças consideradas significativas, foi verificado que quase todos os resultados de comparação dos dias foram inferiores, ou seja, significativos. Entendese assim que houve diferenças entre as médias das pressões trabalhadas.

A diferença mais explícita foi quando comparado o dia 20/09 com os demais dias, possivelmente pelo fato de os equipamentos ainda estarem sendo adaptados aos locais de medição. Outra característica verificada é que os valores de pressão permanecem praticamente o dia todo, em níveis muito elevados. Desse modo, essa região também pode apresentar valores de consumo mais baixos, o que implica no aumento de pressão na rede. Outra característica desse pon- to que pode ter causado alguma diferença dos outros é o fato de ter sido necessária a troca do medidor, pois o primeiro apresentou problema na medição.

O Nó 51 apresentou resultados diferentes dos dois Nós anteriores, sendo que poucas comparações entre dias foram significativas. Assim como os outros, a comparação do dia 20/09 foi a que mais ficou abaixo desse valor. E outros dois dados também foram inferiores; visualizando os resultados obtidos e os dados originais para estudo, visualizamos uma possível alteração nos dias 23/09 e 24/09, que possivelmente foram dias de alguma interferência na rede, para manutenção. Para esse Nó, portanto infere-se que ele tenha valores de pressões bem similares uma à outra e pressões com valores baixos.

Nos Nós 111, 109, 116 e 112 podemos verificar diversos valores de $t$ significativos. Esses nós pertencem a uma mesma região do bairro Vale dos Pinheiros; essa região em geral apresenta valores de pressões médias em relação aos dois outros bairros. As diferenças das médias foram verificadas em maior parte quando se compara o dia 20 com os demais dias.

Isso pode ter ocorrido por ser o primeiro dia de coleta de informação ou por se tratar de um sábado. Como essa região possui algumas pousadas e um hotel, aumenta o fluxo de pessoas, provocando uma alteração no consumo e em consequência a diminuição na pressão, o que acaba causando uma diferença em relação aos demais dias. Um valor que chamou atenção foi a comparação do dia 20 com o dia 24: em 3 dos 4 nós o valor ficou abaixo de 0,005, o que representa uma variação bem significativa das médias encontradas. 
Tabela 3 - Identificação das diferenças significativas entre médias das pressões por nós e dias, com a aplicação do Teste t de Student.

\begin{tabular}{|c|c|c|c|c|c|c|c|c|c|}
\hline & & Sábado & Domingo & Segunda-Feira & Terça-Feira & Quarta-Feira & Quinta-Feira & Sexta-Feira & Sábado \\
\hline NÓ & & $20 / 09 / 2014$ & $21 / 09 / 2014$ & $22 / 09 / 2014$ & $23 / 09 / 2014$ & $24 / 09 / 2014$ & $25 / 09 / 2014$ & $26 / 09 / 2014$ & $27 / 09 / 2014$ \\
\hline \multirow{8}{*}{105} & $20 / 09 / 2014$ & - & 0,159 & 0,010 & 0,035 & 0,035 & 0,037 & 0,125 & 0,395 \\
\hline & $21 / 09 / 2014$ & 0,159 & - & 0,167 & 0,127 & 0,105 & 0,126 & 0,037 & 0,437 \\
\hline & $22 / 09 / 2014$ & 0,010 & 0,167 & - & 0,045 & 0,068 & 0,046 & 0,134 & 0,392 \\
\hline & $23 / 09 / 2014$ & 0,035 & 0,127 & 0,045 & - & 0,024 & 0,002 & 0,092 & 0,406 \\
\hline & $24 / 09 / 2014$ & 0,035 & 0,105 & 0,068 & 0,024 & - & 0,023 & 0,069 & 0,413 \\
\hline & $25 / 09 / 2014$ & 0,037 & 0,126 & 0,046 & 0,002 & 0,023 & - & 0,091 & 0,406 \\
\hline & $26 / 09 / 2014$ & 0,125 & 0,037 & 0,134 & 0,092 & 0,069 & 0,091 & - & 0,430 \\
\hline & $27 / 09 / 2014$ & 0,395 & 0,437 & 0,392 & 0,406 & 0,413 & 0,406 & 0,430 & - \\
\hline \multirow{8}{*}{14} & $20 / 09 / 2014$ & - & 0,028 & 0,011 & 0,008 & 0,007 & 0,645 & 0,019 & 0,017 \\
\hline & $21 / 09 / 2014$ & 0,028 & - & 0,005 & 0,008 & 0,009 & 0,005 & 0,003 & 0,001 \\
\hline & $22 / 09 / 2014$ & 0,011 & 0,005 & - & 0,000 & 0,003 & 0,011 & 0,009 & 0,006 \\
\hline & $23 / 09 / 2014$ & 0,008 & 0,008 & 0,000 & - & 0,001 & 0,013 & 0,011 & 0,009 \\
\hline & $24 / 09 / 2014$ & 0,007 & 0,009 & 0,003 & 0,001 & - & 0,014 & 0,012 & 0,010 \\
\hline & $25 / 09 / 2014$ & 0,645 & 0,005 & 0,011 & 0,013 & 0,014 & - & 0,002 & 0,004 \\
\hline & $26 / 09 / 2014$ & 0,019 & 0,003 & 0,009 & 0,011 & 0,012 & 0,002 & - & 0,002 \\
\hline & $27 / 09 / 2014$ & 0,017 & 0,001 & 0,006 & 0,009 & 0,010 & 0,004 & 0,002 & - \\
\hline \multirow{8}{*}{51} & $20 / 09 / 2014$ & - & 0,149 & 0,006 & 0,024 & 0,042 & 0,645 & 0,665 & 0,120 \\
\hline & $21 / 09 / 2014$ & 0,149 & - & 0,144 & 0,171 & 0,111 & 0,730 & 0,759 & 0,032 \\
\hline & $22 / 09 / 2014$ & 0,006 & 0,144 & - & 0,030 & 0,036 & 0,653 & 0,668 & 0,114 \\
\hline & $23 / 09 / 2014$ & 0,024 & 0,171 & 0,030 & - & 0,066 & 0,634 & 0,653 & 0,142 \\
\hline & $24 / 09 / 2014$ & 0,042 & 0,111 & 0,036 & 0,066 & - & 0,657 & 0,687 & 0,080 \\
\hline & $25 / 09 / 2014$ & 0,645 & 0,730 & 0,653 & 0,634 & 0,657 & - & 0,058 & 0,709 \\
\hline & $26 / 09 / 2014$ & 0,665 & 0,759 & 0,668 & 0,653 & 0,687 & 0,058 & - & 0,737 \\
\hline & $27 / 09 / 2014$ & 0,120 & 0,032 & 0,114 & 0,142 & 0,080 & 0,709 & 0,737 & - \\
\hline \multirow{8}{*}{111} & $20 / 09 / 2014$ & - & 0,071 & 0,025 & 0,043 & 0,001 & 0,033 & 0,030 & 0,047 \\
\hline & $21 / 09 / 2014$ & 0,071 & - & 0,096 & 0,113 & 0,072 & 0,103 & 0,042 & 0,024 \\
\hline & $22 / 09 / 2014$ & 0,025 & 0,096 & - & 0,018 & 0,025 & 0,008 & 0,055 & 0,072 \\
\hline & $23 / 09 / 2014$ & 0,043 & 0,113 & 0,018 & - & 0,043 & 0,010 & 0,073 & 0,090 \\
\hline & $24 / 09 / 2014$ & 0,001 & 0,072 & 0,025 & 0,043 & - & 0,029 & 0,031 & 0,048 \\
\hline & $25 / 09 / 2014$ & 0,033 & 0,103 & 0,008 & 0,010 & 0,029 & - & 0,059 & 0,080 \\
\hline & $26 / 09 / 2014$ & 0,030 & 0,042 & 0,055 & 0,073 & 0,031 & 0,059 & - & 0,017 \\
\hline & $27 / 09 / 2014$ & 0,047 & 0,024 & 0,072 & 0,090 & 0,048 & 0,080 & 0,017 & - \\
\hline \multirow{8}{*}{109} & $20 / 09 / 2014$ & - & 0,155 & 0,057 & 0,040 & 0,043 & 0,002 & 0,125 & 0,121 \\
\hline & $21 / 09 / 2014$ & 0,155 & - & 0,102 & 0,189 & 0,115 & 0,153 & 0,033 & 0,037 \\
\hline & $22 / 09 / 2014$ & 0,057 & 0,102 & - & 0,095 & 0,013 & 0,054 & 0,071 & 0,067 \\
\hline & $23 / 09 / 2014$ & 0,040 & 0,189 & 0,095 & - & 0,082 & 0,042 & 0,161 & 0,157 \\
\hline & $24 / 09 / 2014$ & 0,043 & 0,115 & 0,013 & 0,082 & - & 0,033 & 0,084 & 0,080 \\
\hline & $25 / 09 / 2014$ & 0,002 & 0,153 & 0,054 & 0,042 & 0,033 & - & 0,115 & 0,119 \\
\hline & $26 / 09 / 2014$ & 0,125 & 0,033 & 0,071 & 0,161 & 0,084 & 0,115 & - & 0,004 \\
\hline & $27 / 09 / 2014$ & 0,121 & 0,037 & 0,067 & 0,157 & 0,080 & 0,119 & 0,004 & - \\
\hline \multirow{8}{*}{116} & $20 / 09 / 2014$ & - & 0,087 & 0,067 & 0,096 & 0,005 & 0,596 & 0,005 & 0,053 \\
\hline & $21 / 09 / 2014$ & 0,087 & - & 0,150 & 0,177 & 0,092 & 0,573 & 0,083 & 0,035 \\
\hline & $22 / 09 / 2014$ & 0,067 & 0,150 & - & 0,030 & 0,063 & 0,618 & 0,072 & 0,119 \\
\hline & $23 / 09 / 2014$ & 0,096 & 0,177 & 0,030 & - & 0,092 & 0,630 & 0,101 & 0,146 \\
\hline & $24 / 09 / 2014$ & 0,005 & 0,092 & 0,063 & 0,092 & - & 0,569 & 0,009 & 0,058 \\
\hline & $25 / 09 / 2014$ & 0,596 & 0,573 & 0,618 & 0,630 & 0,569 & - & 0,567 & 0,581 \\
\hline & $26 / 09 / 2014$ & 0,005 & 0,083 & 0,072 & 0,101 & 0,009 & 0,567 & - & 0,049 \\
\hline & $27 / 09 / 2014$ & 0,053 & 0,035 & 0,119 & 0,146 & 0,058 & 0,581 & 0,049 & - \\
\hline \multirow{8}{*}{112} & $20 / 09 / 2014$ & - & 0,104 & 0,151 & 0,022 & 0,009 & 0,188 & 0,087 & 0,089 \\
\hline & $21 / 09 / 2014$ & 0,104 & - & 0,238 & 0,083 & 0,096 & 0,268 & 0,018 & 0,017 \\
\hline & $22 / 09 / 2014$ & 0,151 & 0,238 & - & 0,170 & 0,159 & 0,042 & 0,224 & 0,225 \\
\hline & $23 / 09 / 2014$ & 0,022 & 0,083 & 0,170 & - & 0,013 & 0,206 & 0,066 & 0,067 \\
\hline & $24 / 09 / 2014$ & 0,009 & 0,096 & 0,159 & 0,013 & - & 0,184 & 0,078 & 0,080 \\
\hline & $25 / 09 / 2014$ & 0,188 & 0,268 & 0,042 & 0,206 & 0,184 & - & 0,246 & 0,256 \\
\hline & $26 / 09 / 2014$ & 0,087 & 0,018 & 0,224 & 0,066 & 0,078 & 0,246 & - & 0,002 \\
\hline & $27 / 09 / 2014$ & 0,089 & 0,017 & 0,225 & 0,067 & 0,080 & 0,256 & 0,002 & - \\
\hline
\end{tabular}


O Complot (gráfico de caixa) é elaborado com base em cinco dados estatísticos (Valor mínimo, Primeiro quartil (Q1), Mediana (segundo quartil Q2), Terceiro quartil (Q3) e Valor máximo). A partir dos dados é feito um resumo em forma de gráfico. Esse gráfico é utilizado para avaliar a distribuição empírica dos dados. Por ele também são determinados valores discrepantes, também conhecidos como outliers, que são representados nos gráficos por asteriscos (*).

Os pontos mais altos indicam valores máximos de pressão (no nosso caso), e os valores mais baixos indicam valores mínimos. As caixas fazem referência ao primeiro e terceiro quartis, e a li- nha na metade da caixa indica o segundo quando quartil ou mediana.

Na Fig. 4 vemos o gráfico gerado do ponto 105 . Destaca-se nele que todos os dados de média de pressão registrados estão acima do valor de $60 \mathrm{mca}$. Temos como destaque um outlier no dia 25/09 onde o valor obtido ficou abaixo de $50 \mathrm{mca}$. Observa-se também que no dia 21/09/2014 obteve-se a maior mediana e a menor variabilidade, enquanto as menores medianas são observadas nos dias 20/09/2014 e 24/09/2014. As maiores variabilidades das pressões são observadas nos dias 22/09/2014 e 23/09/2014.

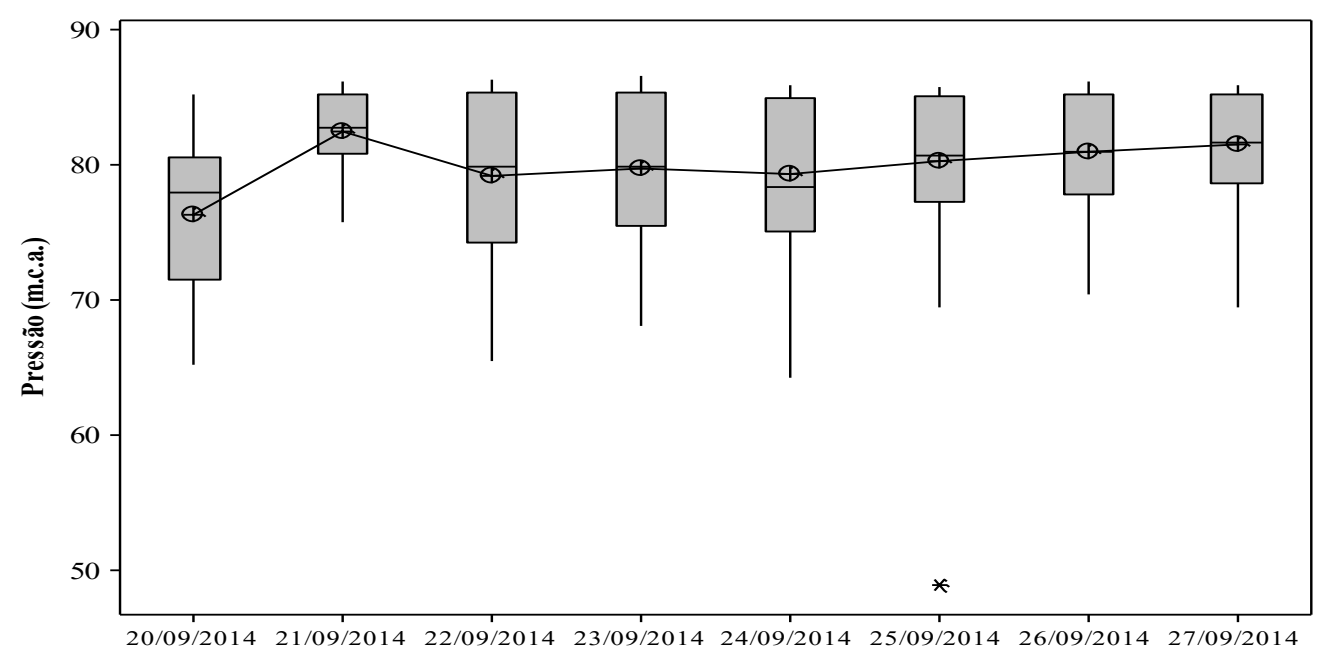

Figura 4 - Complot ponto 105

Na Fig. 5 podemos observar o gráfico do ponto 14. Esse gráfico, diferente do anterior, demonstra que os valores para esse ponto não tiveram grande variabilidade e em geral ficaram acima de 75 mca e abaixo de 85 mca. Destaca-se nesse ponto a existência de pelo menos um outliers em todos os dias da semana, exceto no dia 23/09. Os valores de medianas ficaram todos entre 80 e $85 \mathrm{mca}$, sendo o maior valor apresentado nos dias 24/09 e 27/09. A maior variabilidade foi no dia 23/09, e a menor aconteceu no dia 25/09. 


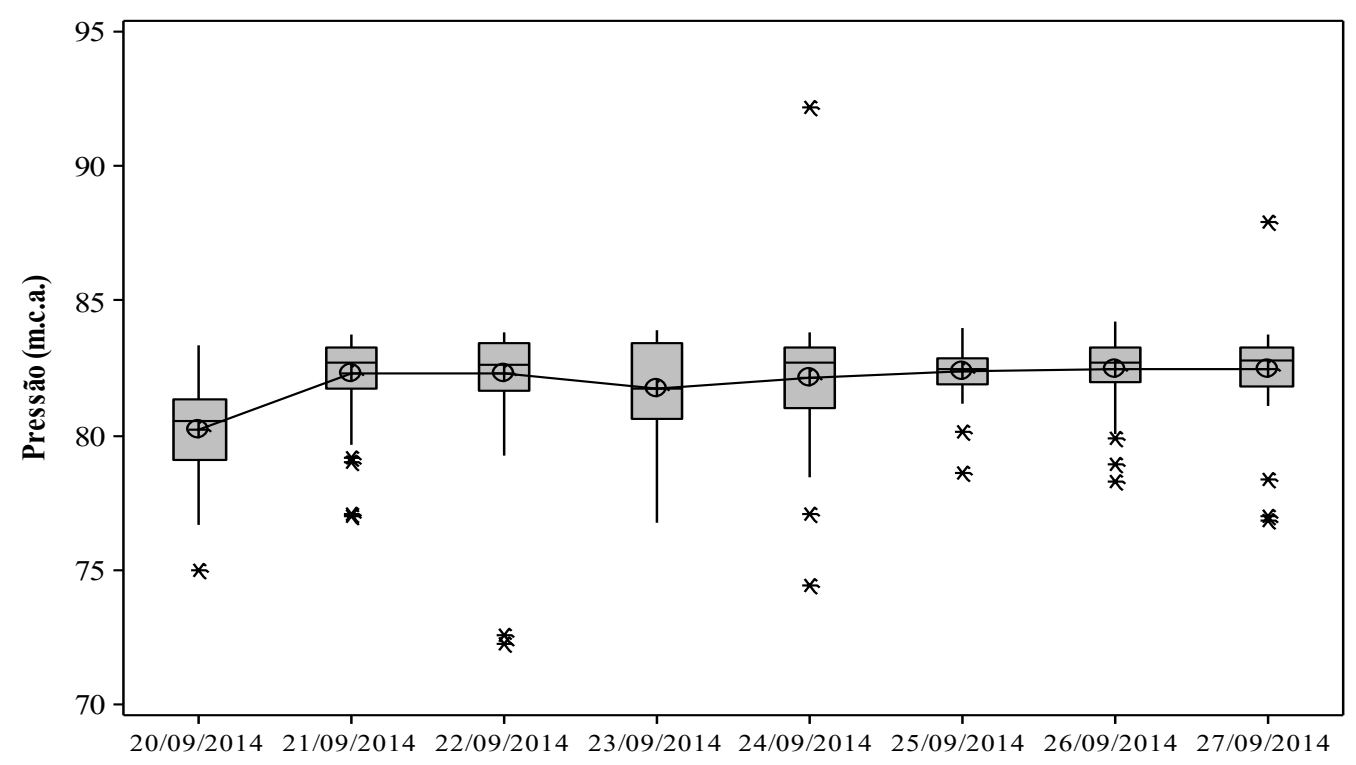

Figura 5 - Complot ponto 14

No gráfico da Fig. 6, temos os dados do ponto 51. Observamos diferentes valores de variabilidades nos dias registrados, sendo todos os dias com pressões abaixo de $50 \mathrm{mca}$. Um destaque é o dia 25/09, que variou entre 20 e 48 mca, demonstrando um comportamento diferente para esse dia com baixas e médias pressões. No dia 26/09 observamos um valor de mediana abaixo de 25 mca, diferenciando e muito dos valores encontrados para os demais dias. A menor variabilidade foi no dia 21/09: seus valores de quartis ficaram entre 45 e 48 mca. Outra coisa que chama atenção em relação aos outros pontos de medição é que nesse local não foi identificado outliers de pressão, mostrando uma configuração análoga em toda a semana.

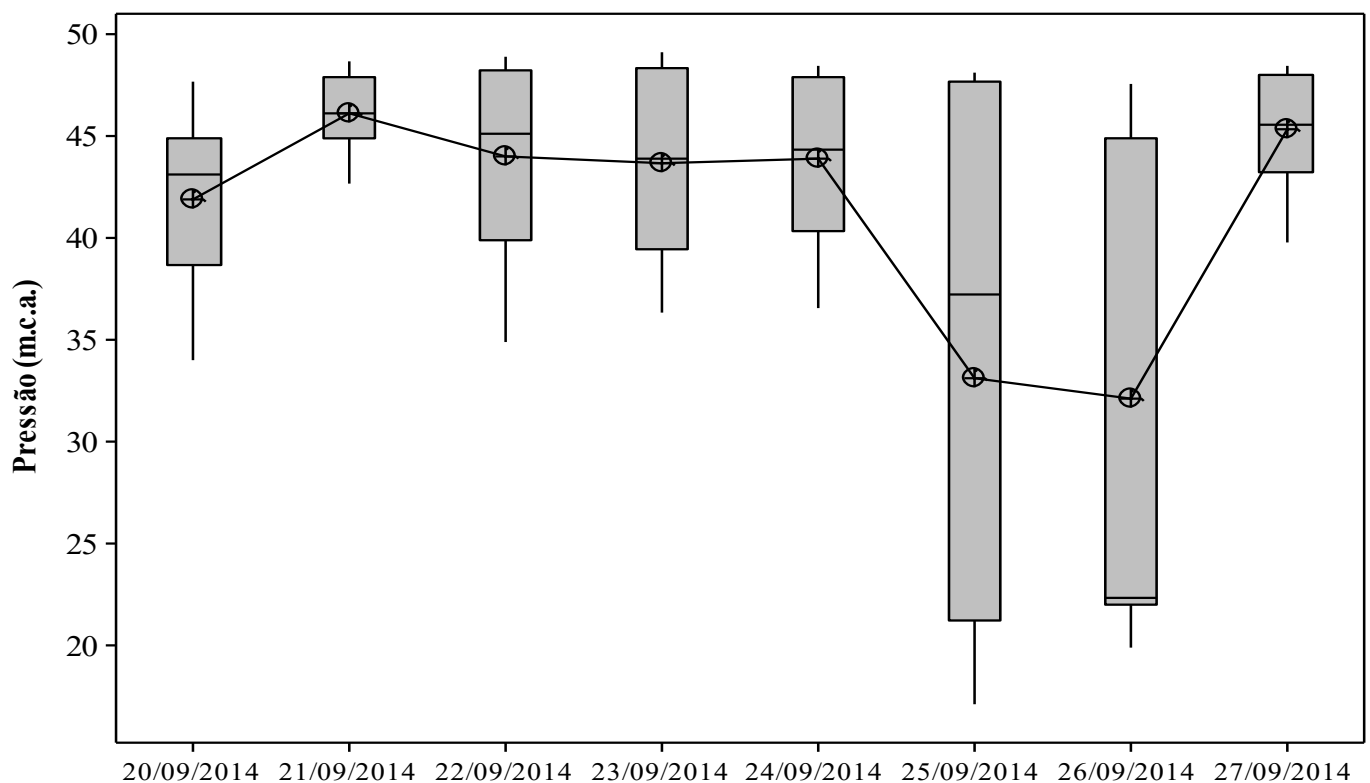

Figura 6 - Complot ponto 51 
O gráfico de boxplot do ponto 111 é demonstrado na Fig. 7. Nele podemos observar que ao longo dos dias obtivemos grande variabilidade dos pontos; somente no dia 21/09 a variabilidade foi menor. Os valores de pressão para esse ponto ficaram entre 75 e 92,5 mca, valor alto para pressão na rede. A menor mediana encontrada foi no dia 20/09, sendo seu valor acima de $85 \mathrm{mca}$. A pressão mínima ocorreu em dois dias (22/09 e 25/09) onde o valor foi de $75 \mathrm{mca}$. Todos os valores de pressão máxima registradas de acordo com o gráfico ficaram acima de 90 mca. Observamos também que não aconteceram outliers nesse ponto.

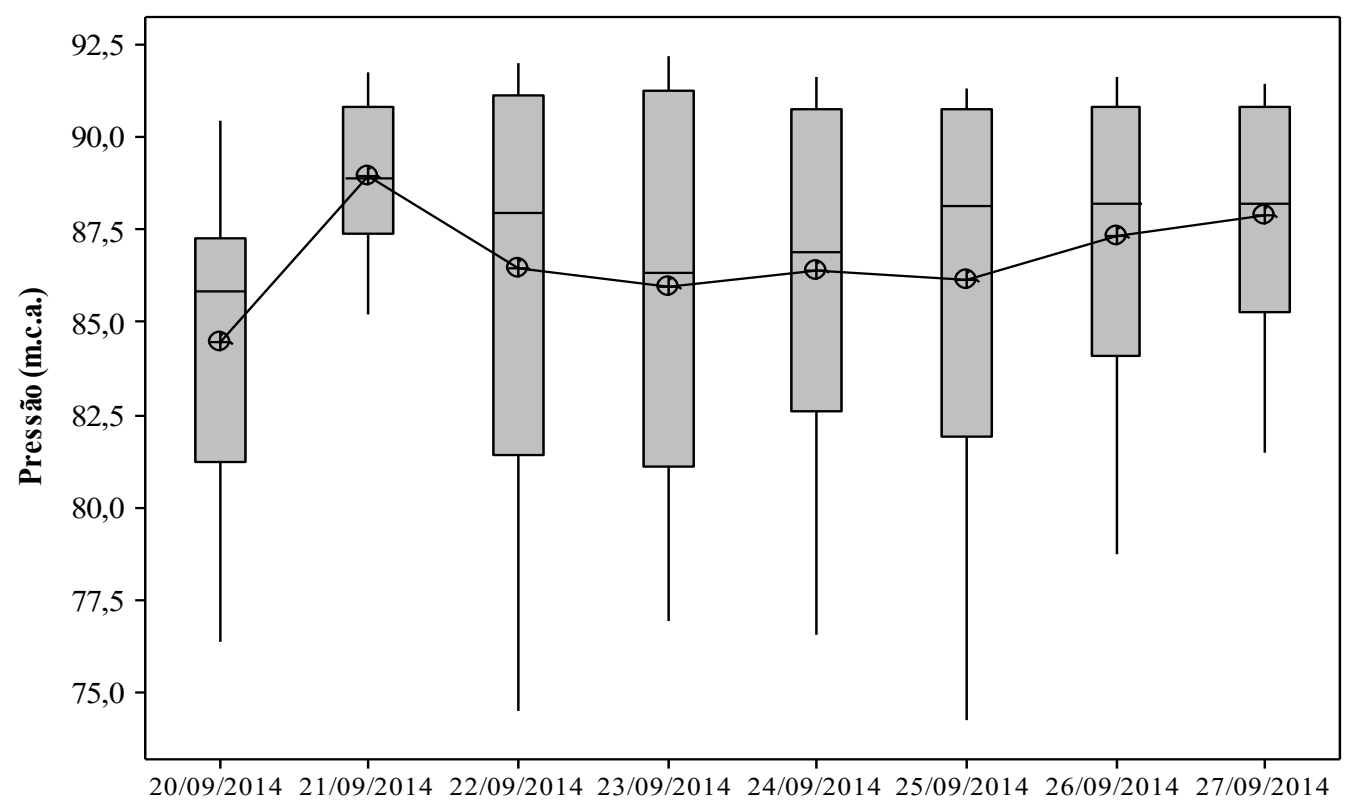

Figura 7 - Complot ponto 111

Na Fig. 8 temos os gráficos do ponto 109. Nele identificamos vários outliers, em todos os dias da semana registraram valores discrepantes. Seus valores de pressão em geral ficaram entre 60 mca e $80 \mathrm{mca}$, isso sem considerarmos os outliers. A variabilidade nesse ponto não foi significativa. A menor variabilidade encontrada foi no dia 21/09, e a maior nos dias 22/09 e 23/09. Todas as medianas ficaram próximas ao valor de 75 mca.
O gráfico Complot para o ponto 116, demonstrado na Fig. 9, apresenta valores de pressão todos abaixo de $45 \mathrm{mca}$. A variabilidade encontrada não foi grande. Os valores de forma geral, desconsiderando os outliers, ficaram entre $25 \mathrm{e}$ 45 mca. A maior variabilidade aconteceu no dia 23/09, e a menor no dia 21/09. Registrou-se como destaque um grande número de outliers no dia $25 / 09$, todos abaixo de $30 \mathrm{mca}$, inferindo a esse dia alguma influência na rede. 


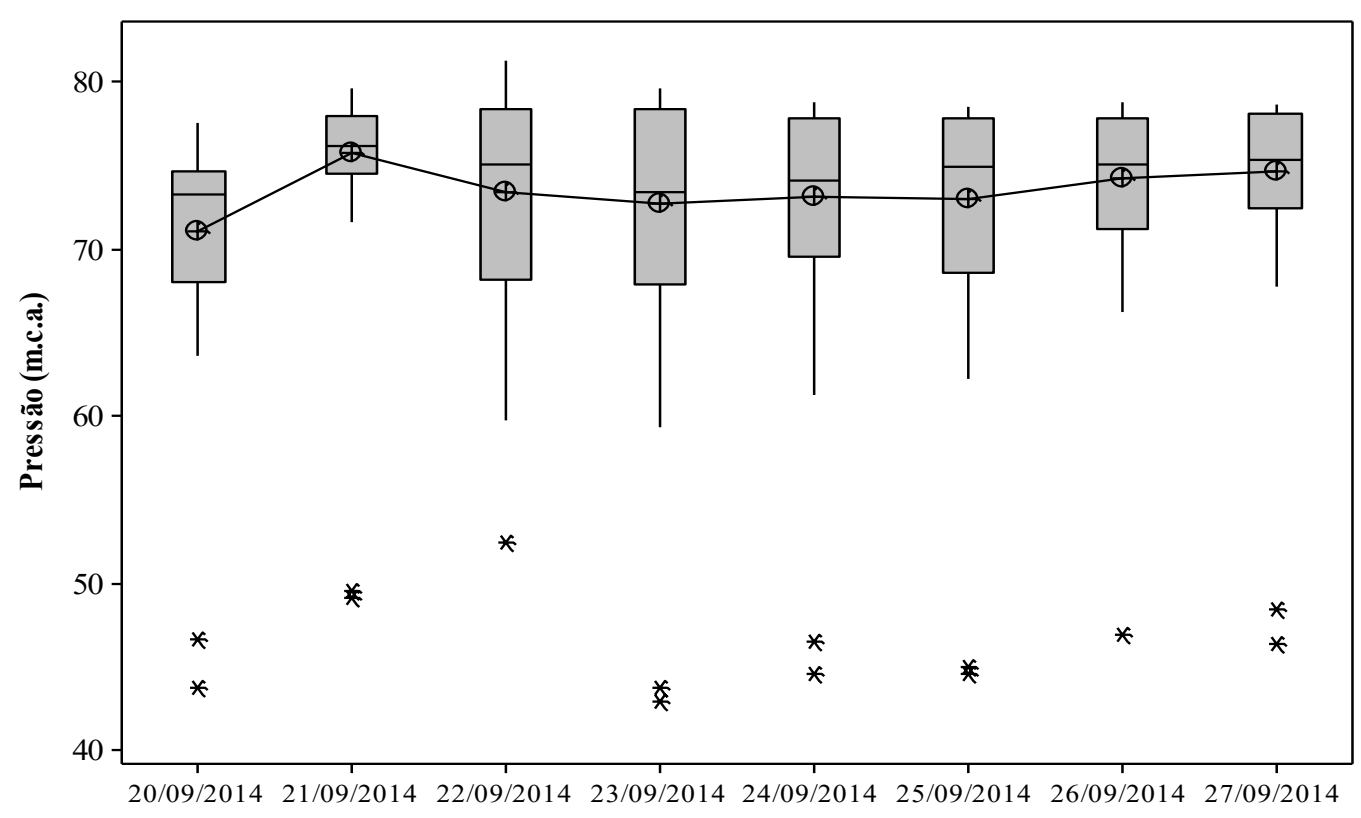

Figura 8 - Complot ponto 109

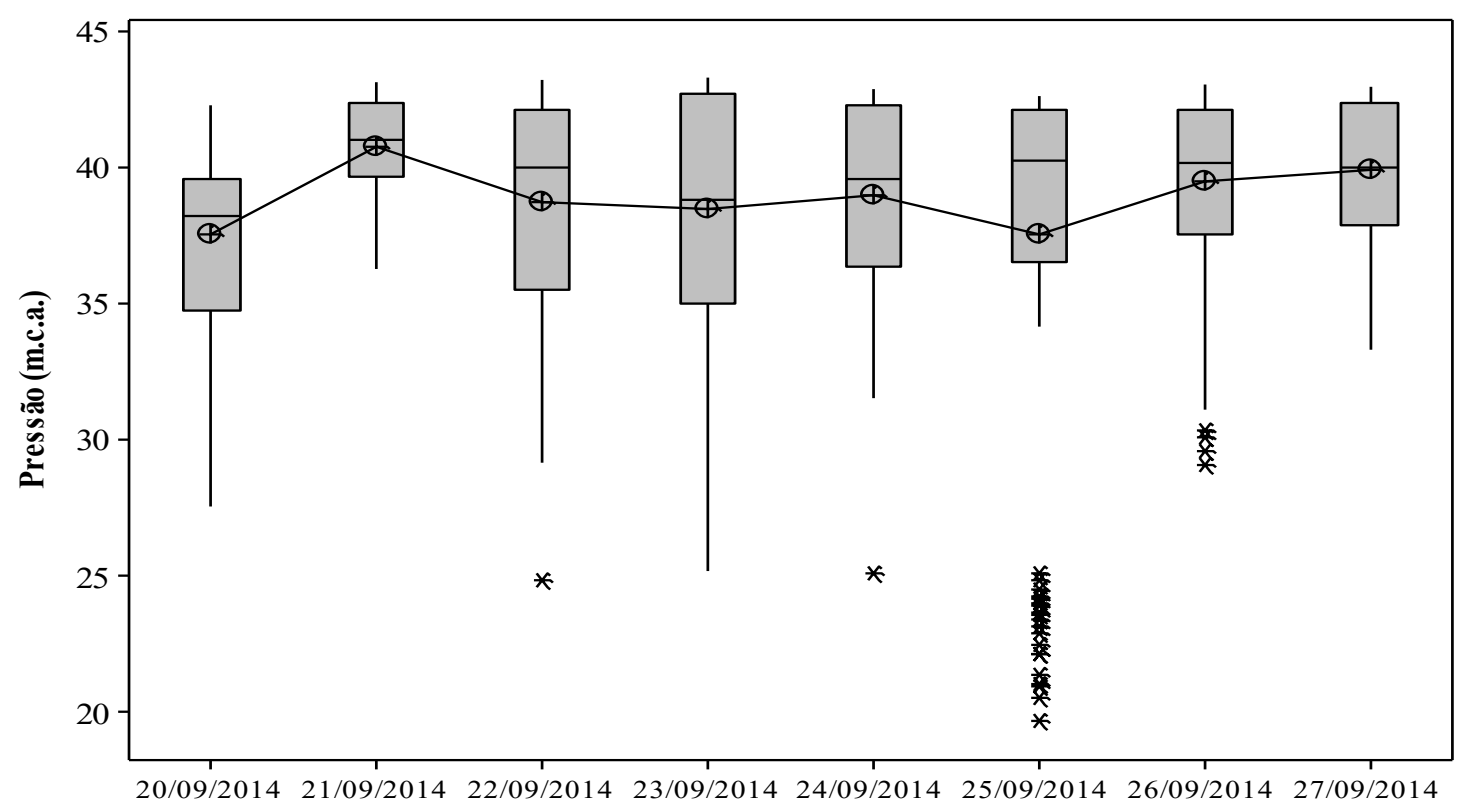

Figura 9 - Complot ponto 116

Na Fig. 10, referente ao gráfico do ponto 112, vemos que a variabilidade das pressões ao longo da semana foi pequena, os valores ficaram todos entre 55 e 65 mca. A menor variabilidade encontrada foi no dia 21/09. As medianas ficaram todas acima de 60 mca. Um destaque desse ponto foi a presença de outliers em quase todos os dias, principalmente no dia 25/09, com alguns valores abaixo de 30 mca. 


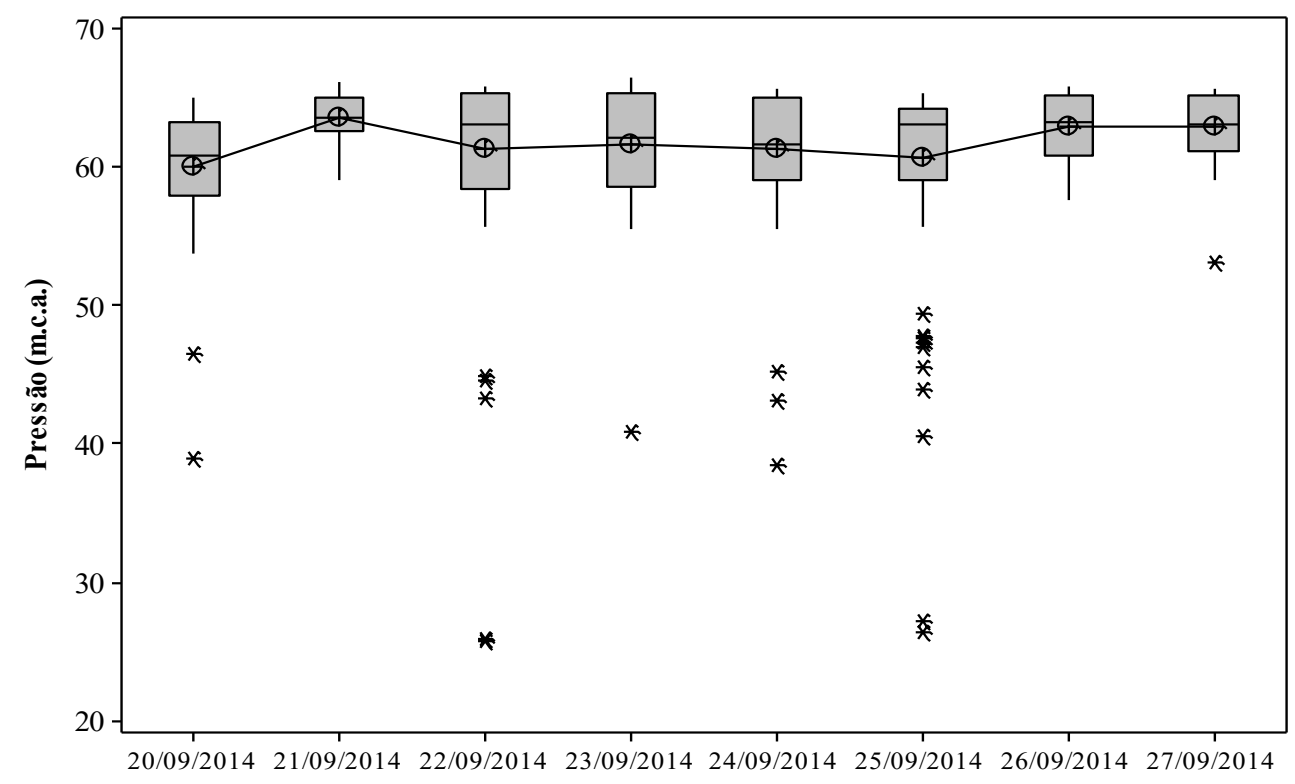

Figura 10 - Complot ponto 112

As perdas em um sistema de distribuição de água implicam diretamente na eficiência da distribuição e comercialização do volume tratado. Diagnosticar os volumes de perdas em determinado setor de distribuição de água proporciona uma melhora no planejamento, investimentos, manobras e manutenção na rede, sem causar maiores prejuízos.

Obteve-se para o valor de perda mínima de 35\% o limite de pressão, que é 23,18 mca, e para o va- lor de perda máxima 65\%; a pressão limite é de 79,94 mca. A partir desses dados foram calculadas as perdas por nós da rede e verificado quais deles obtinham a pressão média fora dos limites estabelecidos.

$\mathrm{Na}$ Fig. 7, foi observado que os pontos 105, 104 e 111 possuem a pressão acima do valor limite e em consequência seus valores de perdas ficaram acima de $65 \%$.

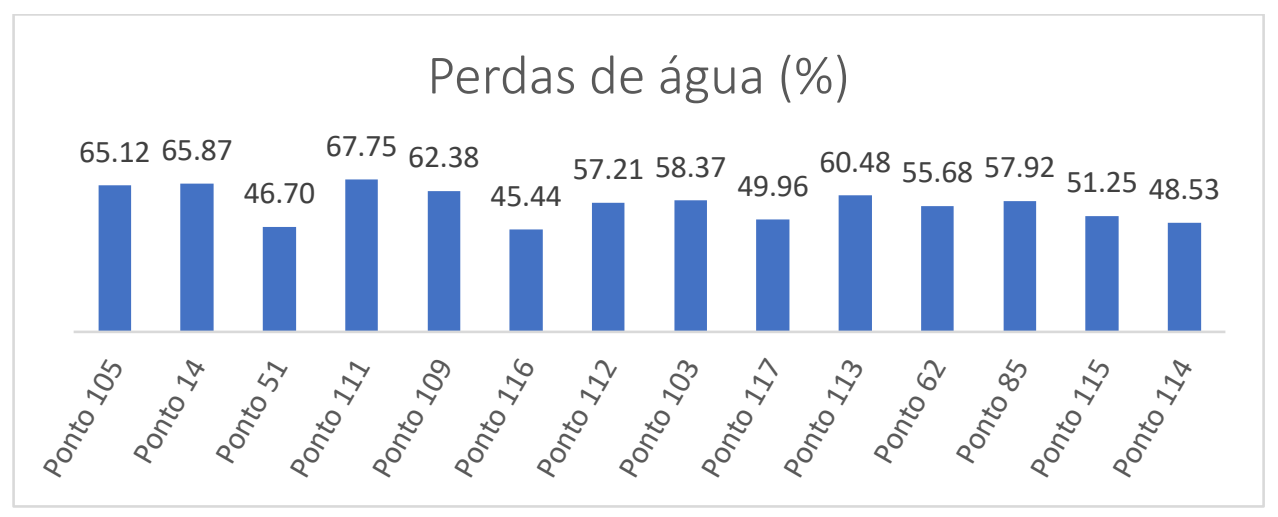

Figura 11 - Gráfico das perdas por Nós

Pode-se observar que nenhuma das perdas ficou abaixo do limite mínimo de $35 \%$. Todas as perdas estão acima de $45 \%$; destaca-se como maior índi- ce de perda o nó 111. Esse ponto está localizado na cota altimétrica mais baixa da rede, no bairro do Vale dos Pinheiros, o que indica que esse ponto 
precisa ser mais estudado e que manobras para diminuição de perdas nesse local serão necessárias. $O$ nó 116 teve a menor perda, está localizado na cota altimétrica mais alta do sistema, pertencente ao bairro Vale dos Pinheiros. Assim, podemos concluir que quanto mais baixa a localização do ponto maior será a pressão e em consequência maior também será o valor de perdas na rede.

\section{CONCLUSÕES}

Após analisar os dados de sete dias consecutivos, conclui-se que a rede se comporta dentro dos padrões esperados. Os finais de semana de maneira geral apresentaram valores de pressão maiores, pois aos sábados e domingos o consumo de água é menor. Já durante a semana de segunda a sexta-feira o valor de pressão é menor, pois o consumo é maior. Esse comportamento foi verificado em todos os Nós estudados.

Foi verificado que os valores maiores de pressão ao longo da semana ficam entre $0 \mathrm{~h}$ e $7 \mathrm{~h}$ da manhã. Como praticamente todas as pessoas estão dormindo nesse intervalo de tempo o consumo é bastante reduzido.

Colocando como parâmetro o valor de pressão na máxima admitida na norma de sistema de distribuição de água que é de $50 \mathrm{mca}$, têm-se alguns valores abaixo e outros acima. Os valores acima na maioria das vezes extrapolam $80 \mathrm{mca}$, aumentando a chance de rompimento das tubulações.

Em relação aos bairros pertencentes ao setor, foi verificado que no bairro Palmela, por possuir cotas altimétricas menores, a pressão é maior, e por se tratar de um bairro com pessoas de menorvalor aquisitivo o consumo de água é menor, talvez por terem residências simples com menos aparelhos que possam consumir mais água. $\mathrm{O}$ bairro Vale dos Pinheiros é pertencente a um condomínio fechado e possui maior valor de consumo por obterem equipamentos que consomem mais; já sua a pressão média é a menor, pois está localizada em postos mais altos da rede.

Houve resultados mais relevantes no que concerne à diferença significativa de valores de pressão para alguns nós com valores mais significativos. 0 nó 14 e o nó 115 são alguns deles, pois tiveram grande variância ao longo dos dias da semana. Isso pode ser causado por alguma manobra significativa na rede. Assim como os nós 51 e 111, que também vieram a apresentar diversos valores significativos durante os cálculos, estes pertencem a uma região com valores mais elevados de cotas altimétricas. Já o ponto 14 e o 105 pertencem a uma área mais baixa da rede no bairro Palmela.

Nos gráficos Complot elaborados, foiidentificado que o dia 21/09 (domingo) em todos os nós foi o que obteve menor variabilidade nos valores de pressão. Já o dia 25/09 (quinta-feira) na maioria dos nós foi o que teve maior variabilidade, exceto em alguns casos. Observou-se também vários outliers; o ponto que chamou mais atenção foi o nó 116, dia 25/09 quando apresentou diversos outliers, diferentemente dos demais dias. Também foram observados valores discrepantes no ponto 14,112 e no 109 em todos os dias.

Com base nesses resultados é possível determinar quais pontos requerem uma maior atenção, e estudar quais seriam as alternativas para melhorar o sistema. Como a pressão está diretamente ligada às perdas de água, o estudo detalhado de análise de pressões impacta diretamente nos estudos de eficiência hídrica e energética. As variações de pressões ao longo das horas e dias da semana podem auxiliar no suporte de estudos e auxiliar gestões em decisões.

\section{AGRADECIMENTOS}

À Redecope Finep - MCT (0983/10), pelo projeto de Desenvolvimento de tecnologias e procedimentos eficientes para gestão hidroelétrica em sistemas de 
abastecimento de água. Os autores também agradecem à Fapemig pelo Programa Pesquisador Mineiro PPM- 00755-16 and PPM - 0633-11. A primeira autora agradece a bolsa da Capes nº 1736715.

\section{CONTRIBUIÇÃO DOS AUTORES}

Todos os autores contribuíram de forma igualitária.

\section{REFERÊNCIAS}

BOBSIN, D. Estudo de demanda hídrica em sistema de abastecimento de água na cidade de São Lourenço - MG. 112 p. Dissertação (Mestrado) - Universidade Federal de Itajubá, UNIFEI, Itajubá, 2016.

CAMBRAINHA, G. M. G.; FONTANA, M. E. Análise da aplicação de investimentos em perdas de água no nordeste brasileiro. Revista Eletrônica em Gestão, Educação e Tecnologia Ambiental, Santa Maria, v. 19, n. 2, p. 983-994, mai./ago. 2015. https://doi. org/105902/2236117015520

FONTANA, N.; GIUGNI, M.; PORTOLANO, D. Losses reduction and energy production in water-distribution networks. Journal of water resources planning and management, Reston, v. 138, n. 3 , p. 237-244, May 2012. https://doi.org/10.1061/(ASCE)WR.19435452.0000179

FORMIGA, K. T. M.; CHAUDHRY, F. H. Modelos de análise hidráulica de redes de distribuição de água considerando demanda dirigida pela pressão e vazamentos. Revista engenharia sanitária e ambiental, Rio de Janeiro, v. 13, n. 2, p. 153-162, abr./jun. https:// doi.org/10.1590/S1413-41522008000200005

FORTES, L. R. Análise de sistema de distribuição de água com a aplicação de balanço hídrico energético. 82 p. Dissertação (Mestrado) - Universidade Federal de Itajubá, UNIFEI, Itajubá, 2016.

GOMES, H. P. Sistemas de Saneamento - Eficiência Energética. 1. Ed. João Pessoa: Editora Universitária/UFPB, 2010. 1ª Edição. 366 p. ISBN 978-85-7745-607-9

GUEDES, H. A. S.; SILVA, D. D.; ELESBON, A. A. A.; RIBEIRO, C. B. M.; MATOS, A. T.; SOARES, J. H. P. Aplicação da análise estatística multivariada no estudo da qualidade da água do Rio Pomba, MG. Revista Brasileira de Engenharia Agrícola e Ambiental, Campina Grande, v. 16, n. 5, p. 558-563, fev. 2012. https://doi.org/10.1590/ S1415-43662012000500012

GUIDI, J. M. M. Análise da variação de consumo de água utilizando dados obtidos por sistemas supervisores remotos - estudo de caso: município de Franca - SP. 92 p. Dissertação (Mestrado) Universidade Estadual de Campinas, UNICAMP, Campinas, 2016.
KANAKOUDIS, V. et al. A new set of water losses-related performance indicators focused on areas facing water scarcivty conditions. Journal Desalination and Water Treatment, Balaban, V. 51, n. 13-15, april 2013. https://doi.org/10.1080/19443994.2012.748448

LAMOGLIA, H. A. Estudo da correlação entre perdas de água e energia para setores do sistema de abastecimento de água da cidade São Lourenço - MG. 237 p. Dissertação (Mestrado) Universidade Federal de Itajubá, UNIFEI, Itajubá, 2013.

MELO, L. D. V. Aplicação de técnicas estatísticas para avaliação de desempenho e confiabilidade de estações de tratamento de água. 110 p. Dissertação (Mestrado) - Universidade Federal de Minas Gerais, UFMG, Belo Horizonte, 2014.

MUTIKANGA, H. E.; SHARMA, S. K.; VAIRAVAMOORTHY, K. Methods and tools for managing losses in water distribution systems. Journal of water resources planning and management, Reston, v. 139, n. 2, p. 166-174, Mar. 2013. https://doi.org/10.1061/(ASCE) WR.1943-5452.0000245

PINNTO, M. R.; CASTRO, M. A. H.;BARBOSA, J.M. C.; MAIAJUNIOR,J.V. F. Dimensionamento econômico otimizado de redes de distribuição de água considerando custos de manutenção. Revista engenharia sanitária e ambiental, Rio de Janeiro, v. 22, n. 1, p. 145-153, jan./ fev. 2017. https://doi.org/10.1590/S1413-41522016140349

REIS, E.; MELO, P.; ANDRADE, R.; CALAPEZ, T. Estatística aplicada. Lisboa: Edições Sílabo, 2015.

RIBEIRO, D. L. C. Estudo de calibração da rede de distribuição de água de São Lourenço - MG. 191 p. Dissertação (Mestrado) Universidade Federal de Itajubá, UNIFEI, Itajubá, 2014.

RODRIGUES, J. A. O impacto da utilização de diferentes equipamentos de medição no índice de perdas em um setor do sistema de abastecimento de água da cidade de Campo Grande - MS. 183 p. Dissertação (Mestrado) - Fundação Universidade Federal de Mato Grosso do Sul, UFMS, Campo Grande, 2014.

SANTOS, J. Estudo de Modelo de Calibração para ajuste de parâmetros de rugosidade, demanda e vazamento visando eficiência hídrica e energética em redes de abastecimento de água. Estudo de caso em São Lourenço - MG. 185 p. Dissertação (Mestrado) - Universidade Federal de Itajubá, UNIFEI, Itajubá, 2016.

SILVA, F.G.B. Estudos de calibração de redes de distribuição de água através de algoritmos genéticos. $234 \mathrm{f}$. Tese (Doutorado em Hidráulica e Saneamento) - Escola de Engenharia de São Carlos, Universidade de São Paulo, São Carlos, SP, 2003.

TUCCIARELLI, B. T.; CRIMINISI, A.; TERMINI, D. Leak analysis in pipeline systems by means of optimal valve regulation. Journal of hydraulic engineering, [S.I], v. 22, n. 1, p. 277-285, march. 1999. https://doi.org/10.1061/(ASCE)0733-9429(1999)125:3(277)

VIEIRA, L.T.S. Análise de comportamentos de parâmetros hidráulicos de uma rede de distribuição de água do sul de Minas Gerais. Dissertação (Mestrado em Meio Ambiente e Recursos Hídricos), Universidade Federal de Itajubá, 2019. 